Ágora Rev. Cient. 2017; 04(02):e6

\title{
Factores asociados al abuso sexual en niños atendidos en la Comisaría La Huayrona de San Juan de Lurigancho, 2017
}

\author{
Factors associated with sexual abuse in children attended at the La Huayrona Commissioner of San \\ Juan de Lurigancho, 2017
}

Olga Rea Abad ${ }^{1 \mathrm{a}}$, Yeny Pariona Paquiyayrin ${ }^{1 \mathrm{a}}$, Nadezhda Arias Romani ${ }^{1 \mathrm{~b}}$

RESUMEN

Objetivo: Determinar los factores asociados al abuso sexual en niños atendidos en la Comisaria la Huayrona de San Juan de Lurigancho, 2017. Materiales y Métodos: El estudio fue cuantitativo, retrospectivo, nivel descriptivo, diseño no experimental-transversal. La muestra estuvo conformada por 30 padres de familia de los niños víctimas a quienes se aplicó previamente el consentimiento informado. Resultados: Se evidencia que, en el factor familiar, del total de padres, el $40 \%$ son conyugues separados así mismo el $46.7 \%$ de casos es cometido por un familiar. Factor socioeconómico: el $80 \%$ de los conyugues trabajan, 46,6\% tienen un ingreso económico bajo (s/ 300.00 - 600.00 soles). Factor cultural se evidencia que el $80 \%$ de las madres toman las decisiones en casa por ausencia del padre. El 46.7\% de madres en el hogar es víctima de violencia física. Conclusiones: Respecto al factor familiar la mayoría de conyugues son separados, los niños quedan al cuidado de familiares cercanos, factor socioeconómico la mayoría de padres trabajan y sus ingresos económicos son bajos entre s/300.00 a 600.00 soles. Respecto al factor cultural asociado al abuso sexual en la mayoría de casos quien toma las decisiones en casa es la madre ya que hay ausencia del padre por motivo de violencia familiar.

Palabras clave: abuso sexual, violación, factor familiar, factor socioeconómico, factor cultural.

\begin{abstract}
Objective: To determine the factors associated with sexual abuse in children assisted at the Huayrona Police Station in San Juan de Lurigancho, 2017. Materials and Methods: The study was quantitative, retrospective, descriptive level, non-experimental-transversal design. The sample consisted of 30 parents of the children of victims to whom informed consent was previously applied. Results: It is evident that, in the family factor, of the total number of parents, $40 \%$ are separated spouses, while $46.7 \%$ of cases are committed by a family member. Socioeconomic factor: $80 \%$ of spouses work, $46.6 \%$ have a low economic income (S/ 300.00 600.00 soles). Cultural factor shows that $80 \%$ of mothers make decisions at home due to the father's absence. $46.7 \%$ of mothers in the home are victims of physical violence Conclusions: Regarding the family factor, most spouses are separated, children are left in the care of close family members, the majority of parent's work, and their income is low. S/ 300.00 to 600.00 soles. Regarding the cultural factor associated with sexual abuse in most cases who makes decisions at home is the mother since there is absence of the father due to family violence.
\end{abstract}

Keywords: sexual abuse, rape, family factor, socioeconomic factor, cultural factor.

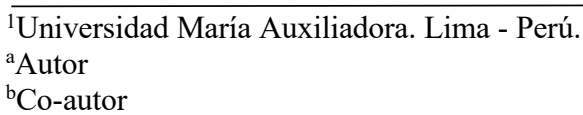

\section{INTRODUCCIÓN}

A nivel mundial, según UNICEF, la violencia infantil ha llegado a extremos nunca antes vistos, en los cuales el asesinato es sólo la punta del iceberg. En 58 países más de la mitad de los pequeños son disciplinados mediante violencia en sus casas o en las escuelas, y en países como
Tanzania, Kenia o Zimbabue 1 de cada 3 niñas y 1 de cada 6 niños ha sufrido alguna forma de abuso sexual. Entretanto en países más desarrollados como Estado Unidos, casi un 25\% de las adolescentes y un $10 \%$ de los chicos ha sufrido algún tipo de incidente sexual, abuso, robo $\mathrm{o}$ asalto (1). 
A nivel Latinoamérica, en el año 2012, de acuerdo con la Encuesta Nacional de Victimización Delictiva de la Oficina de Estadísticas Judiciales, se denunciaron 346,830 violaciones o agresiones sexuales de personas menores de 12 años de edad o más (2).

El suceso sexual una vez ocurrido produce daños físicos y psicológicos al menor, provocando un fuerte impacto emocional, lesiones graves, alteraciones psicológicas y de conducta sexual, alterando también sus relaciones con otras personas, produciendo a su vez la ausencia de denuncias por parte del abusado, que en la mayoría de los casos entra en un estado de negación, pérdida de autoestima, y hasta a veces un extraño sentimiento de ambivalencia hacia el abusador abordado de sentimientos como el miedo y agresividad.

Dicha problemática va incrementando día tras día en el Perú, según el ministerio de la mujer y poblaciones vulnerables (MIMP) entre enero y setiembre de este año, se han registrado 356 abusos sexuales en menores, ocupando el tercer lugar en casos de violación (3).

En el distrito San Juan de Lurigancho, la incidencia de casos de violación sexual cada vez cobra mayor importancia las edades más vulnerables se encuentran entre 7 a 17 años, según el anuario de la PNP en el año 2016 se registraron 1004 denuncias de violación sexual todas del sexo femenino y en la actualidad las cifras se han incrementado, el problema más grave para el menor no es solo que se cometa contra él este delito, sino que el menor no avise que ocurrió por el contrario se aislé con el hecho cometido y sus hechos, creyendo que es el único culpable. Cabe resaltar que el abuso sexual está relacionado con una serie de factores vinculados a elementos culturales, familiares y socioeconómicos que, actuado de manera independiente o en conjunto, configuran condiciones de riesgo para los menores.

\section{MATERIALES Y MÉTODOS}

El estudio realizado considera el carácter cuantitativo, retrospectivo y descriptivo, además de emplear el diseño no experimental y transversal. La población estuvo conformada por el total de padres de los niños víctimas de abuso sexual entre las edades de 1 a 14 años en el año 2016 hasta el mes de junio del año 2017. Trabajando con la población total (30 casos) $\mathrm{La}$ técnica empleada en el presente trabajo de investigación consideró la encuesta. Además, como instrumento se empleó el cuestionario elaborado con base en las 3 dimensiones: familiar, socioeconómico y cultural. Para la validación de los instrumentos de la recolección de datos se realizó por revisión de expertos según las características de calidad, objetividad, actualidad, organización, suficiencia, intencionalidad, consistencia, coherencia, metodología, pertinencia. La confiabilidad fue establecida en base a la recolección de datos a partir de una muestra piloto, cuyos resultados fueron sometidos a la prueba Alfa de Cronbach. Fueron considerados principios éticos, incluyendo el consentimiento informado para los participantes del estudio. Los datos obtenidos fueron analizados por medio del programa estadístico e informático SPSS.

\section{RESULTADOS}

En la Tabla 1 se representa el factor familiar asociado al abuso sexual se observa que el 50,0\% son familias funcionales dentro de ellos "casados" evidenciando el respeto entre sus miembros, una comunicación adecuada, se apoyan unos a otros, enfrentan los conflictos con una adecuada madurez, para promover un desarrollo favorable para todos sus miembros. Asimismo, se representa el parentesco con el agresor. Se observa que el 46,7 de los encuestados respondieron "familiar" en la mayoría de casos, este acto es cometido por familiares directos (tíos, padrastros).

Tabla 1. Factor familiar asociado al abuso sexual, según estado conyugal de los padres y parentesco con el agresor.

\begin{tabular}{lccllll}
\hline \multicolumn{1}{c}{$\begin{array}{c}\text { Estado } \\
\text { conyugal }\end{array}$} & $\mathbf{N}^{\circ}$ & $\%$ & & $\begin{array}{c}\text { Parentesco } \\
\text { con el } \\
\text { agresor }\end{array}$ & $\mathbf{N}^{\mathbf{0}}$ & $\%$ \\
\cline { 4 - 6 } $\begin{array}{l}\text { Casado } \\
\text { Separado }\end{array}$ & 15 & 50,0 & Familiar & 14 & 46,7 \\
& 12 & 40,0 & Amigo & 6 & 20,0 \\
& 3 & 10.0 & Vecino & 3 & 10,0 \\
Otros & & & Desconocido & 7 & 23,3 \\
& & & & & &
\end{tabular}

Total $30 \quad 100,0$ $30 \quad 100,0$

En la Tabla 2, se representa el factor socioeconómico asociado al abuso sexual se observa que el $80,0 \%$ de los encuestados respondieron "ambos padres trabajan "siendo así los menores quedan muchas veces solos o al cuidado de familiares: abuela, tíos, vecinos. Asimismo, se observa que el ingreso económico en el hogar es bajo "S/ 300.00-600.00" con el $46,6 \%$. 
Tabla 2. Factor socioeconómico asociado al abuso sexual, según situación laboral de los padres e ingreso económico.

\begin{tabular}{lcccc}
\hline $\begin{array}{l}\text { Situación laboral de los } \\
\text { padres }\end{array}$ & $\mathbf{N}^{\mathbf{0}}$ & $\mathbf{\%}$ & $\begin{array}{l}\text { Ingreso } \\
\text { económico }\end{array}$ & $\begin{array}{l}\mathbf{N}^{\circ} \\
\%\end{array}$ \\
\hline Ambos padres trabajan & 24 & 80,0 & $300-600$ & 14 \\
Solo el padre trabaja & 2 & 6,7 & $600-900$ & $\begin{array}{l}46,7 \\
10\end{array}$ \\
Solo la madre trabaja & 0 & 0,0 & $900-1200$ & 63,3 \\
No trabajan & 4 & 13,3 & & 20,0 \\
\hline \multicolumn{1}{c}{ Total } & $\mathbf{3 0}$ & $\mathbf{1 0 0 , 0}$ & $\mathbf{3 0}$ \\
\hline
\end{tabular}

En la Tabla 3 se representa el factor cultural asociado al abuso sexual se observa que el $40 \%$ de los encuestados respondieron "Mamá" en la mayoría de casos en el hogar la que dirige y delega las actividades y deberes a realizase es la madre. Seguido por "Papá y Mamá" 33,3\% ambos conyugues toman las decisiones en el hogar estando en un nivel medio. Asimismo, el 20,0\% corresponde a un solo conyugue "padre" en un nivel bajo y por último corresponde a "hermanos"
$6,7 \%$. Así mismo el $66.7 \%$ de madres en el hogar es víctima de violencia física.

Tabla 3. Factor cultural asociado al abuso sexual según, toma de decisiones en casa y violencia familiar.

\begin{tabular}{lcclll}
\hline $\begin{array}{l}\text { Toma de decisiones } \\
\text { en casa }\end{array}$ & $\mathbf{N}^{\circ}$ & $\%$ & $\begin{array}{c}\text { violencia } \\
\text { familiar }\end{array}$ & $\mathbf{N}^{\circ}$ & $\%$ \\
\hline Papá & 6 & 20,0 & física & 20 & 66,7 \\
Mamá & 12 & 40,0 & psicológica & 10 & 33,3 \\
Hermanos & 2 & 6,7 & & & \\
Papá y mamá & 10 & 33,3 & & & \\
\hline Total & $\mathbf{3 0}$ & $\mathbf{1 0 0 , 0}$ & $\mathbf{3 0}$ & $\mathbf{1 0 0 , 0}$ \\
\hline
\end{tabular}

En la Tabla 4 se representa los factores asociados al abuso sexual se observa que en el factor familiar la mayoría de los conyugues son "casados" con el $50 \%$ y según el parentesco es un "familiar" $46,7 \%$. Respecto al factor socioeconómico se evidencia que la mayoría de padres "trabajan" $80 \%$ pero tales ingresos son bajos con un porcentaje de 46,7. Respecto al factor cultural quien toma las decisiones en el hogar es la "madre" con un $40 \%$.

Tabla 4. Factores asociados al abuso sexual en niños atendidos en la Comisaria La Huayrona de San Juan de Lurigancho, 2017

\begin{tabular}{|c|c|c|c|c|c|c|c|c|c|c|c|c|c|c|c|c|c|}
\hline $\begin{array}{l}\text { Estado } \\
\text { conyugal }\end{array}$ & $\mathrm{N}^{\circ}$ & $\%$ & $\begin{array}{c}\text { Parent } \\
\text { agresor }\end{array}$ & $\mathrm{N}^{\mathrm{a}}$ & $\%$ & $\begin{array}{l}\text { Situac } \\
\text { laboral }\end{array}$ & $\mathrm{N}^{\circ}$ & $\%$ & $\begin{array}{l}\text { Ingreso } \\
\text { económico }\end{array}$ & $\mathrm{N}^{\circ}$ & $\%$ & Toma decis & $\mathrm{N}^{\circ}$ & $\%$ & $\begin{array}{l}\text { Violencia } \\
\text { familiar }\end{array}$ & $\mathrm{N}^{\circ}$ & $\%$ \\
\hline Conviviente & 8 & 26,7 & Familiar & 14 & 46,7 & Ambos trabajan & 24 & 80.0 & $300-600$ & 14 & 46,7 & Papá & 6 & 20,0 & Física & 20 & 66.7 \\
\hline Casado & 7 & 23,3 & Amigo & 6 & 20,0 & Solo el padre & 2 & 6,7 & $600-900$ & 10 & 33,3 & Mamá & 12 & 40,0 & Psic & 10 & 1033,3 \\
\hline Separado & 12 & 40,0 & Vecino & 3 & 10,0 & Solo la madre & 0 & 0,0 & $900-1200$ & 6 & 20,0 & Hnos & 2 & 6,7 & & & \\
\hline Otros & 3 & 10,0 & Descon & 7 & 23,3 & No trabajan & 4 & 13.3 & & & & Papá y mamá & 10 & 33,3 & & & \\
\hline Total & 30 & 100,0 & & 30 & 100,0 & & 30 & 100, & & 30 & 100,0 & & 30 & 100,0 & & 30 & 100,0 \\
\hline
\end{tabular}

\section{DISCUSIÓN}

En relación al objetivo general, determinar los factores asociados al abuso sexual en niños atendidos en la Comisaria la Huayrona de San Juan de Lurigancho, 2017. Se evidencia que los factores asociados que determinan el abuso sexual en niños son el factor familiar, el factor socioeconómico y factor cultural.

Según el factor familiar asociado al abuso sexual, el 50,0\% de las parejas tienen la condición de "casados", familias funcionales evidenciando el respeto entre sus miembros, una comunicación adecuada, se apoyan unos a otros, enfrentan los conflictos con una adecuada madurez, para promover un desarrollo favorable para todos sus miembros.

Del mismo modo es señalado en el estudio realizado por Alape Z. (4) indica que el agresor es en la mayoría de los casos es un individuo conocido por la familia. A pesar de no especificar el parentesco, puede asumirse que se trata de un miembro de la familia.

Según el factor familiar en el caso de familias disfuncionales donde se caracteriza por falta de empatía y poca compresión dentro del hogar generando conflictos y discusiones. No comparten momentos juntos ya que cada uno está metido en sus asuntos personales, ausencia del rol que cada padre debe cumplir, y por último las contradicciones de los padres en cuanto a la educación de sus hijos. Según Yáñez. (5) sustenta que en los hogares disfuncionales el problema y los desacuerdos familiares conlleva a un riesgo de los menores dentro y fuera del hogar, estando propensos a ser víctimas de abuso sexual.

Según el factor socioeconómico asociado al abuso sexual el $80,0 \%$ de padres de los niños 
sometidos al abuso trabajan "siendo así los menores quedan muchas veces solos o al cuidado de familiares cercanos. Asimismo, el ingreso familiar oscila entre $\mathrm{S} / 300.00$ a 600.00 soles que contribuye al abandono del niño.

Del mismo modo es señalado en el estudio. Morales J. (6) indica que los problemas económicos conllevan a tener un estado de salud física y mental con sentimientos de tristeza, soledad y de ansiedad entrando a un cuadro de desesperación lo cual lo lleva a abusar del niño.

Según el factor cultural asociado al abuso sexual el $80,0 \%$ de las familias que dirigen el hogar son las madres, quienes, al pasar mayor tiempo con los hijos, no existe la figura paterna lo que genera un estado de vulnerabilidad. Según la teoría de Freud (7) indica el complejo de emociones y sentimientos infantiles caracterizados por la presencia simultánea y ambivalente de deseos amorosos y hostiles hacia los progenitores, este aspecto conlleva a que los cuidadores e incluso progenitores confunden estos sentimientos de sus hijos y los conlleva a abusar de ellos.

\section{CONCLUSIONES}

Los factores que están asociados al abuso sexual en niños atendidos en la Comisaria $\mathrm{La}$ Huayrona de San Juan de Lurigancho son: factores familiares, factores socioeconómicos y factores culturales. Respecto al factor familiar asociado al abuso sexual, está determinado por familias disfuncionales generando en los menores el descuido en su cuidado. Respecto al factor socioeconómico asociado al abuso sexual en la mayoría de casos los padres trabajan, pero sus ingresos económicos son bajos. Respecto al factor cultural asociado al abuso sexual en la mayoría de casos quien toma las decisiones en casa es la madre ya que hay ausencia del padre por motivo de violencia familiar.

\section{AUTOR DE CORRESPONDENCIA}

Mg. Nadezhda Arias Romaní

Universidad María Auxiliadora

Av. Canto Bello No 431

Lima 36 - Perú

Teléfono: +51-948989657

E-mail: adhzedan@hotmail.com

\section{REFERENCIAS BIBLIOGRÁFICAS}

1. Fondo para la Infancia de las Naciones Unidas (UNICEF). [Online].2017 [citado 3 de marzo del 2017]. Disponible en: http://noticias.universia.es/actualidad/notici a/2014/10/22/1113605/unicef-violenciainfantil-cifras.html

2. Truman, J. L. Langton y M. Planty, "Criminal Victimización 2012", Departamento de Justicia de los Estados Unidos, Oficina de Programas Judiciales, Oficina de Estadísticas Judiciales, octubre de 2013.[online][citado 3 de marzo].Disponible en:

http://www.bjs.gov/content/pub/pdf/cv12.pd $\mathrm{f}$

3. Ministerio de la mujer y poblaciones vulnerables (MIMP), Encuesta Demográfica y de Salud Familiar (ENDES)[online] 2017 [citado 4 de marzo]Disponible en:https://peru21.pe/lima/peru-ocupa-tercerlugar-casos-violacion-sexual-mundo190003

4. Alape C, Castaño J, Castaño L, Abuso sexual y factores asociados en población escolarizada perteneciente a una institución educativa de la ciudad de Manizales, Caldas (Colombia), 2012;13(1):47-60. Archivos de Medicina (Col) [Internet]; 13(1) Disponible en: 2013; http://ridum.umanizales.edu.co:8080/xmlui/ bitstream/handle/6789/84/abuso\%20sexu al\%20ins\%20educativa.pdf?sequence

5. Zunzunegui M. morales J. Martínez V. (10) realizaron un estudio sobre: Maltrato infantil factores socioeconómicos y estado de salud [online]. 2007 [citado el 20 de marzo 2017]. Disponible en: https://www.aeped.es/sites/default/files/anal es/47-1- 7.pdf

6. Viviano T. Abuso sexual: Estadísticas para la reflexión y pautas para el Buen Trato 1ra edición setiembre. (Hecho el depósito legal en la biblioteca nacional del Perú 201264 pág.

7. Werner, Shirli, Malaspina, Dolores y Rabinowitz, Jonathan. Factores socioeconómicos en la familia 18 de abril. [Internet] 2007[citado el 12 de enero]. Disponible en: https://espanol.thelawdictionary.org/factores -socioeconomicos/ 